Department: Applications

Editor: Mike Potel, potel@wildcrest.com

\title{
Measurement and Inspection of Photo-Realistic 3D VR Models
}

Sławomir Konrad Tadeja

Department of Engineering, University of Cambridge, Cambridge, UK

Wojciech Rydlewicz

Centrum Systemów Softdesk, Łódź, Poland

Yupu Lu

Department of Engineering, University of Cambridge, Cambridge, UK

Tomasz Bubas

Centrum Systemów Softdesk, Łódź, Poland

Maciej Rydlewicz

Centrum Systemów Softdesk, Łódź, Poland

Per Ola Kristensson

Department of Engineering, University of Cambridge, Cambridge, UK

Abstract-Recent advancements in virtual reality (VR) may help unlock the full potential offered by 3D photorealistic models generated using state-of-art photogrammetric methods. Using VR to carry out analyses on photogrammetric models has the potential to assist the user in performing basic off-line engineering inspection of digital twins-digitized representations of real-world objects and structures. However, for such benefits to materialize it is necessary to create suitable interactive systems for working with photogrammetric models in VR. To this end this paper presents PhotoTwinVR-an immersive gesture-controlled system for manipulation and inspection of 3D photogrammetric models of physical objects in VR. An observational study with three domain experts validates the feasibility of the system design for practical use-cases involving off-line inspections of pipelines and other 3D structures.

Photogrammetry is a set of methods for extracting information about physical objects from their photographic surveys [1]. Such infor- mation can then be used to construct 3D models, called digital twins, of these real-world objects.

We see great potential in embedding pho- 
togrammetric 3D model manipulation within virtual reality (VR) environments. In particular, an interactive VR system for photogrammetry can provide an effective environment for working with digital twins [2]. A successful realization of such an environment can then provide benefits, such as removing the need for a physical presence in the vicinity of the physical object under investigation. However, to realize this vision it is critical that there exist effective interaction methods for working with photogrammetric models in VR.

This paper addresses this gap in the literature by presenting PhotoTwinVR - an immersive gesture-controlled system for inspection, and dimension measurements of 3D photogrammetric models of real-life structures in VR. The user can move the model to any part of 3D space, decrease or increase the model's size and rotate the model along a chosen axis in $3 \mathrm{D}$ space. This bimanual manipulation method is facilitated by egocentric hand-tracking sensing attached to the front of the VR headset. The user interface also supports taking measurements of the model using a built-in measurement toolkit.

The feasibility of the system design is validated in an observational study with three domain experts using a mixed-methods design. Two of the domain experts work for a Polish state institute, the Urzad Dozoru Technicznego (UDT) ${ }^{1}$.The third expert is an experienced designer of industrial piping systems. The three experts were asked to complete an off-line inspection of a 3D photogrammetric model of a piping system using the measurement toolbox offered by our system. The positive reactions, comments and overall results that emerged from the study suggest that the system was successful in fulfilling its purpose.

\section{Surveying of a Real-World Asset}

The process of carrying out an engineering survey of an existing physical asset demands effort, time, and other, more tangible, resources. For example, it may be desired to measure the height and width of the supporting structure, as well as the distance between two neighboring pylons of a pipe-rack (see Fig. 1). Such a task may prove to be an expensive, time-consuming and cumbersome process. The pipe-rack is spanning

\footnotetext{
${ }^{1}$ eng. Office of Technical Inspection: www.udt.gov.pl
}

over a road with a pedestrian footpath on one side and railings on the other. There is also vegetation present on both sides of the structure. All of these factors would undoubtedly influence the measurement procedures if performed traditionally by, for example, limiting how, and when, someone would be able to start and complete measurements.

To conduct such an engineering survey, the first step would normally be to arrange for scaffolding to be placed around the structure. Even if it is decided to rely on a form of laser-based measuring it would most likely still be required to place scaffolding on at least both sides of the construction. Due to safety concerns, irrespective of which pylons are chosen to put the scaffolding around, parts of the road or pedestrian passage would have to be closed, or part of the present vegetation would have to be cut (see Fig. 1). Further, working at heights has its own associated risks, and such work is also sensitive to weather conditions. All these preparations, potentially closing part, or in its entirety, a highly-used road, and later the time and resources needed for the erection and removal of the scaffolding may render the entire procedure lengthy and costly.

As an alternative, with the help of our system we can obtain the same surveying information from a precise photo-realistic 3D model of the pipe-rack from the safety of an engineering office. By using our PhotoTwinVR system, the user can promptly capture the desired measurements of the structure without the need to be physically near it. The 3D photogrammetric model itself can be generated from image data captured with the help of an unmanned aerial vehicle (UAV), which effectively eliminates the need for scaffolding.

\section{System Architecture}

Since VR is still in its infancy, appropriate interaction techniques and devices for VR are still under active research. Hence, there is no standard set of interaction devices and techniques suitable for all use-cases. Normally, the user cannot comfortably use a computer mouse or keyboard when wearing a VR headset that, by design, completely immerses the user within a virtual world.

\section{Function Model and Task Analysis}

We used Function Analysis System Technique (FAST) [3] to capture the central system 


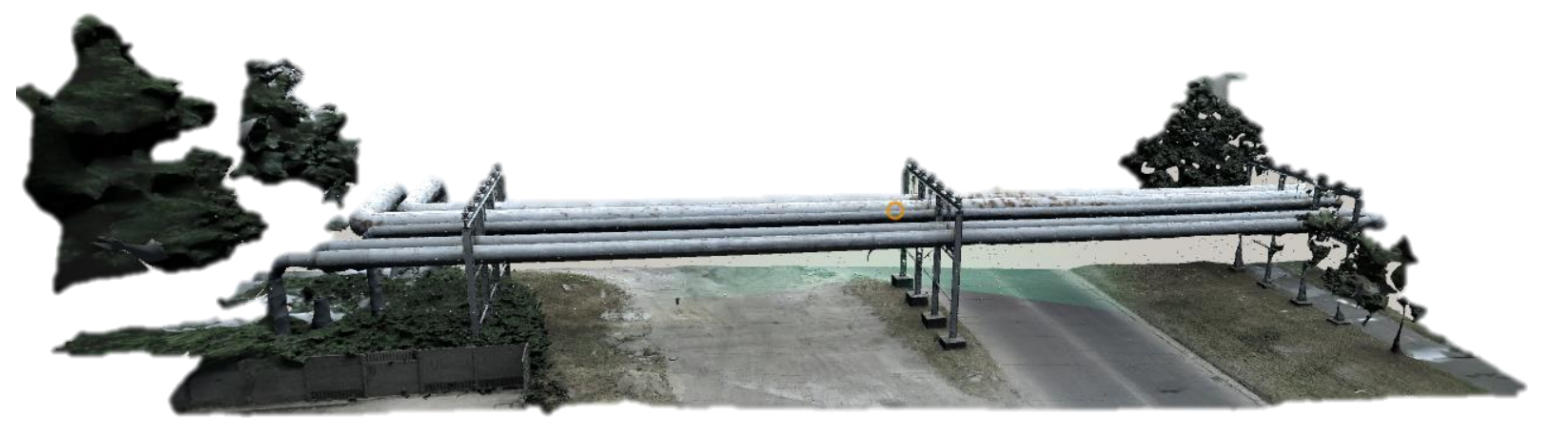

Figure 1. The 3D photogrammetric model of the pipe-rack as seen by the VR user.

requirements. The FAST analysis resulted in the understanding of four main high-level tasks that are required to carry out the overall functions:

T1-Model visualization: The system should allow the user to visualize and interact with any given model representing a 3D object.

T2-Model manipulation: The user should be able to effectively manipulate the visualized model. The manipulation functionality should allow three basic operations: (a) rotation of the 3D model around a chosen axis; (b) displacement of the model within 3D space; (c) changing the size of the model.

T3-Model inspection: The system should allow the user to visually inspect the model.

T4-Model measurement: The system should allow the user to take and retake desired measurements of the model. The measurements should be automatically stored and easily retrievable for future reference.

\section{System Structure}

We observe the way signals flow between the key system elements in order to reason about its overall system structure (Fig. 2).

(A) User: The user exchanges signals with the system in two ways. First, there is a bidirectional signal via the system's depiction of a ray-traced orange cross-hair, which signals to the system what the user is focusing on at the moment. In response, an interactive element is highlighted, thereby signaling that this element is ready to interact with the user.

(B) Hand-tracking: As the main method of interaction is gestural input (see Fig. 3), the signals received and transmitted by this component form a feedback loop. The position of the hands and their gestures are always signaled to the user using virtual hand representations. If the virtual hands are not visible to the user then the physical hands are outside the sensor's tracking area. Actions executed by the user on either the model (C) or the measurement tools (D) are immediately applied, and, as such, signaled to the user using a range of visual clues.

(C) Photogrammetric model: The model (C) responds instantly to all operations initiated by gestural input. These operations include object displacement (Fig. 4), rotation (Fig. 5), and resizing (Fig. 6).

(D) Measurement tools: The measurement toolbox includes the rulers (vectors/lines in 3D space), ruler markers (small, spherical markers), and the legend shown to the user in the heads-up display (HUD) (see Fig. 8-10). All these objects respond to actions invoked with the user's hands. The markers can be created, displaced, removed, and highlighted while the user has selected them using a gaze-controlled cross-hair.

\section{Virtual Reality Environment}

We used the Unity engine to implement and test our system. The hardware setup consisted of the Oculus Rift HMD, its position-tracking sensor, and the Leap Motion hand-tracking sensor. The basis of the VR framework underpinning the system was previously developed for aeroengine digital twin assessment [4] and used in another qualitative user study [5]. This prior user study [5] was concerned with a different photogrammetric model and the participants in that study were chosen due to their expertise in civil engineering rather than piping systems. 
Gaze-Tracking (Cross-Hair)

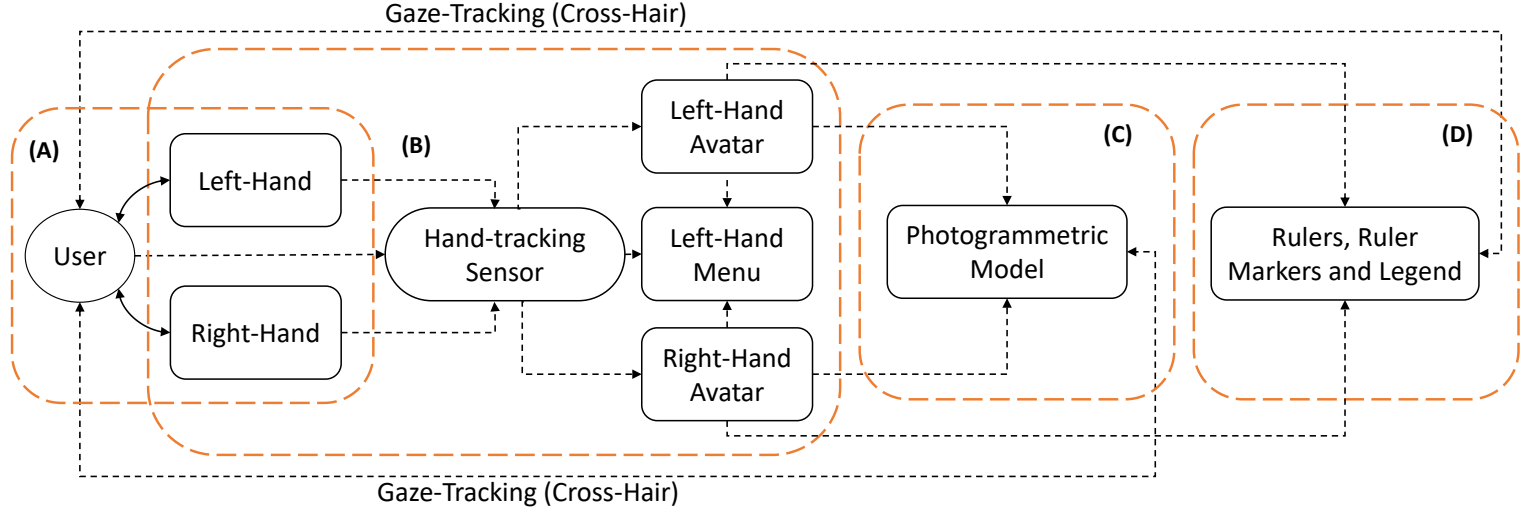

Figure 2. The diagram models the signals flowing between key elements of the visualization system: $(A)$ the user; (B) the hand-tracking setup; $(C)$ the 3D model; and (D) the measurement toolkit. Both unidirectional and bidirectional signals are shown. $(A)$ and $(B)$ overlap as they both rely on hand tracking.

\section{Photogrammetric Model: Pipe-Rack}

The VR environment is demonstrated by being populated with a photo-realistic reconstruction of an existing pipeline system (see Fig. 1). The 3D model represents a pipe-rack installation located in Łódź, Poland.The image data used to create this model was captured by a UAV and was processed by ContextCapture ${ }^{2}$. In ContextCapture, an algorithm to automatically detect pixels which correspond to the same physical points is implemented. Visual data extracted from numerous images allows camera positions to be defined and a precise 3D shape of the physical object and terrain to be generated. The capture process itself is called multi-image close-range digital photogrammetry. The photographs were obtained during a ten minute long UAV flight. The model was reconstructed from 204 photos with a re-projection error (RMS) of only 0.58 pixels and was geo-referenced using photo metadata gathered by the UAV. As a result, the model was properly scaled and geo-positioned. The flight plan was a combination of manual steering with automated mission planning calculated with Drone Harmony ${ }^{3}$.

These particular pipelines are used to transport heating media to residential buildings and nearby industrial facilities. The piping system is supported by three steel frames leading the pipes above the road. The rack's supporting system needs to restrict the deflection caused by gravity

\footnotetext{
${ }^{2}$ www.bentley.com/en/products/brands/contextcapture

${ }^{3}$ droneharmony.com/
}

loads as well as wind and other environmental factors. Such pipelines and supporting frames need to be inspected on a regular basis. With the help of a camera-equipped UAV this inspection can be executed faster and safer, but not necessarily more accurately. However, having a correctly scaled and geo-positioned photogrammetric model enables safer and more accurate off-line inspections.

\section{Interaction Methods: Gaze and Hand Tracking}

Object manipulation is realized using gestural input. The gesture recognition capabilities are based on the Leap Motion SDK. There are four types of gestures that are recognized by the system (see Fig. 3).

\section{Model Manipulation Methods}

The system supports three primary methods manipulating a 3D model: (1) displacement; (2) rotation; and (3) resizing. To be able to manipulate the model, the user presses the [house] button on the left-hand menu (see Fig. 3(a)).

(1) Displacement: The user can move the model into any position by using any hand to execute a pinch gesture to select an object. Next, as the user moves the hand this results in a displacement of the model corresponding to the hand's movement (see Fig. 4). This procedure can be repeated as many times as required to place the model in the desired location.

(2) Bimanual Rotation: When the user makes a 


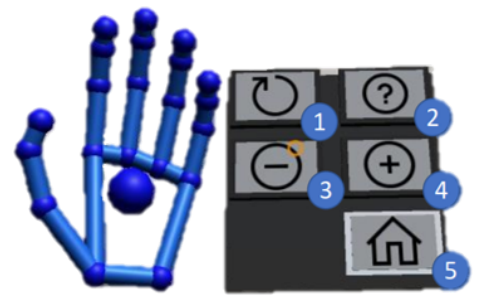

(a)
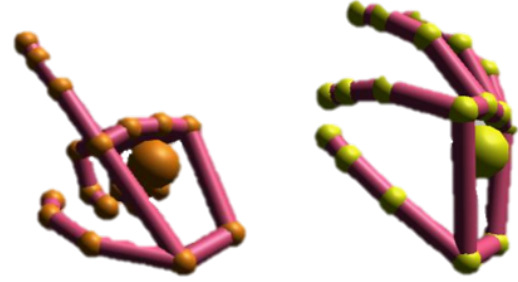

(b)

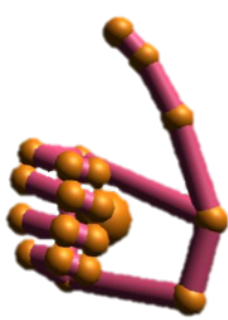

(c)

Figure 3. The avatars of the left-hand (in blue) and the right-hand (in red) respectively. (a) Left-hand palm-up gesture reveals the attached menu: (1) reset visualization; (2) help menu; (3) remove ruler markers; (4) add ruler markers; and (5) allow model manipulation. If no buttons are pressed the user can connect the markers with rulers to take measurements. (b) The pinch gesture (b) is used to move the model in 3D space. The thumbs-up gesture (c) is used to release a marker. Icons courtesy of icons8.com.

pinch gesture with both hands and starts to rotate their hands, one of the hands becomes the rotation axis while the other hand generates the rotation arc (see Fig. 5).

(3) Bimanual Resizing: To scale an object the user first grabs the model using both hands and can thereafter change its size by adjusting the distance between the hands (see Fig. 6).

\section{Snapping Grid}

The primary objective of the snapping grid is to support the user in the process of placing the ruler markers around and on the model. Initially the snapping points, are placed in fixed positions around the model. When the user attempts to place the marker in close vicinity of such a point, the marker automatically snaps to the snapping point.

To generate the grid, we first create a dense 3D snapping grid around the model. Next, we check which snapping points the object collided with and remove all points where no collision is detected. The snapping radius, the distance from which the ruler markers are snapped into position on a snapping point, is automatically scaled when the scale of the model changes.

\section{Measuring the 3D Model}

Taking a measurement, a length between any two points in 3D space, consists of two steps. First, the user presses the $[+]$ button on the lefthand menu (see Fig. 3(a)). Next, the user generates and places at least two markers in the desired locations near the model (see Fig. 8). Finally, the user has to connect the selected markers with the ruler. The length of this ruler, measured from within the centers of the markers, is the Euclidean distance in 3D space shown in the HUD.

\section{Domain Expert Study}

We used a mixed-methods approach with three domain experts and two different models [5] to assess the suitability of the system. After watching a short video clip, the participants were asked to perform slightly different tasks depending on which model they were working with. Here, we report on the results related to the piperack model.

\section{Participants}

All the participants, referred to as P1, P2 and P3, had been either working for the UDT (P1), or reported having years of industrial experience (P2 and P3). P1 was 26 years old and held a degree in power engineering. P2 was 41 years old and held a degree in process engineering and he had worked for six years as a plant pipelines designer. P3 was 29 years old, held an engineering degree in power engineering and had four years of experience as a plant pipeline designer.

\section{Training and Procedure}

The participants reported basic or non-existent prior experience with either VR or hand-tracking. We therefore provided them with some initial training by presenting a brief video clip explain- 


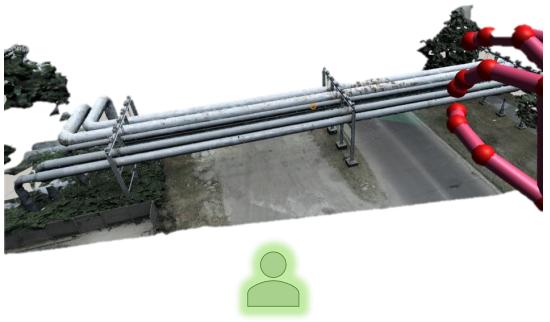

(a)

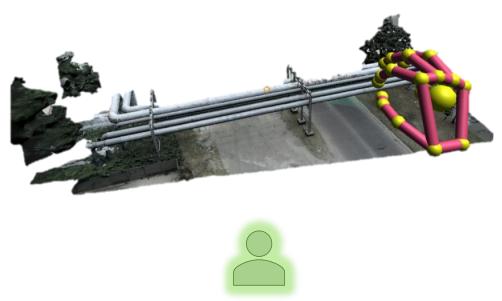

(b)

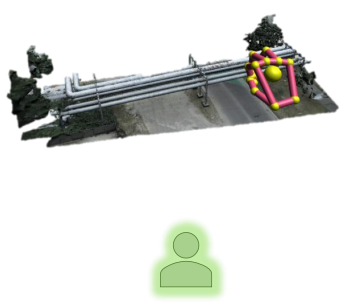

(c)

Figure 4. The user moves the model further down from its current position by (a) grabbing the object with the pinch gesture; and $(b-c)$ extending the grabbing hand forward.

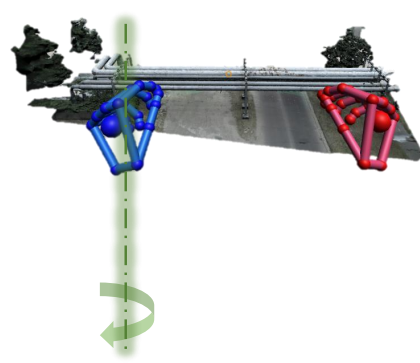

(a)

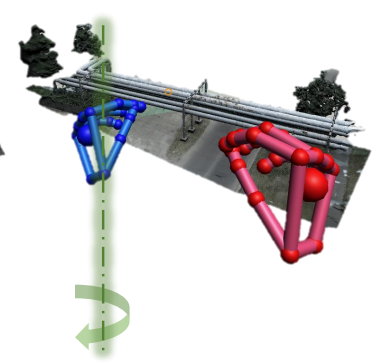

(b)

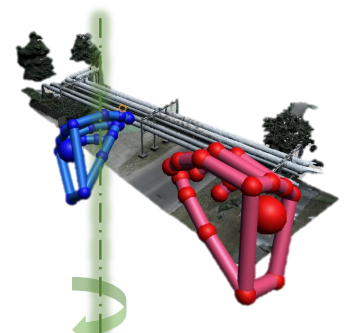

(c)

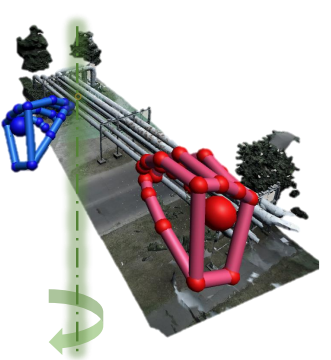

(d)

Figure 5. The user bimanually rotates the model $(a-d)$. The left hand is the rotation axis. We do not explicitly require one hand to be rotation axis as the virtual axis can sit anywhere on the line connecting two pinch points.

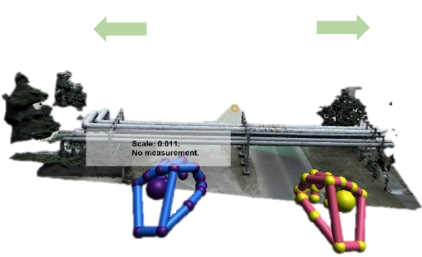

(a)

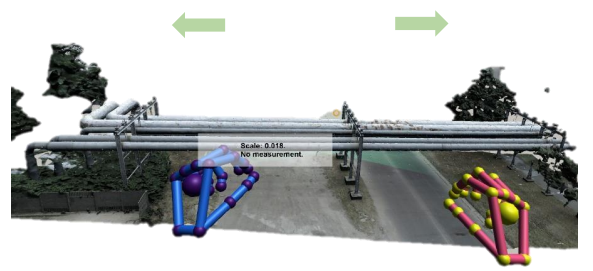

(b)

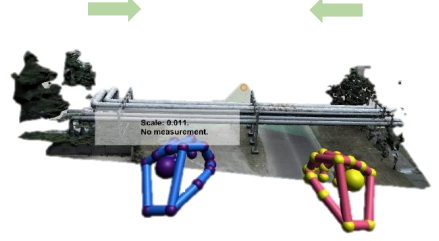

(c)

Figure 6. The user resizes the model by making bimanual pinch gestures and continuously moving the hands apart to enlarge $(a-b)$, or moving them closer together to reduce the size of the model (c). The green arrows show the direction in which the user's hands are moving. The HUD shows the difference in the models' scale.

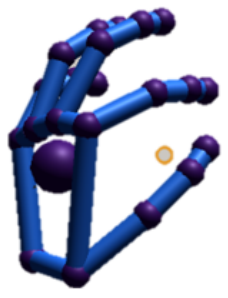

(a)

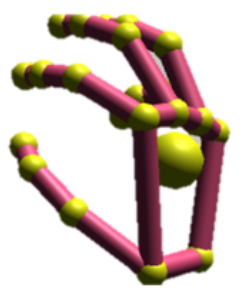

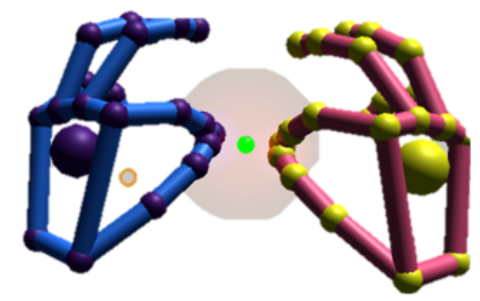

(b)

Figure 7. To generate the new markers the user has to (a) make the pinch gesture with both hands at once, which causes (b) the generation of a new marker.

ing the interaction techniques and the pipe-rack model in the system.
In the study phase the participants first familiarized themselves with the interaction methods 


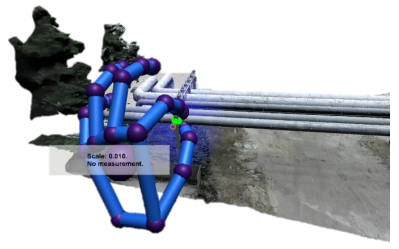

(a)

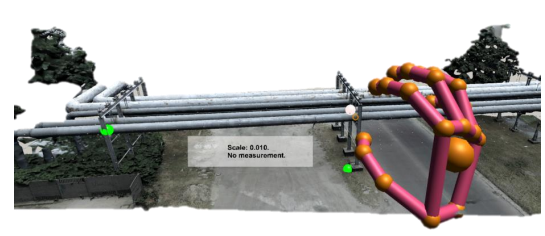

(b)

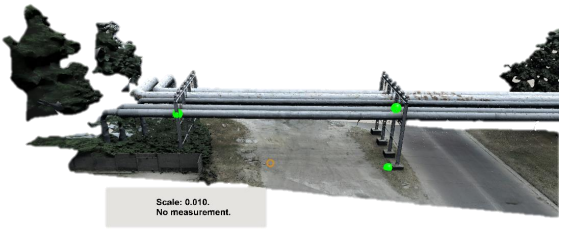

(c)

Figure 8. After the creation of the markers the user can place them anywhere using either hand $(a-b)$. The HUD shows the model's current scale and up to three of the most recent measurements $(b-c)$. To release a marker, the user can use a thumbs-up gesture or wait for it to be automatically deselected after two seconds.

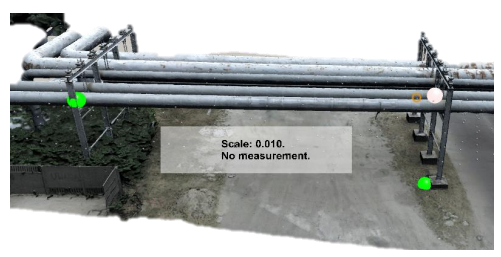

(a)

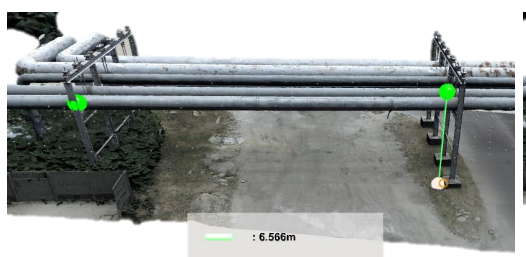

(b)

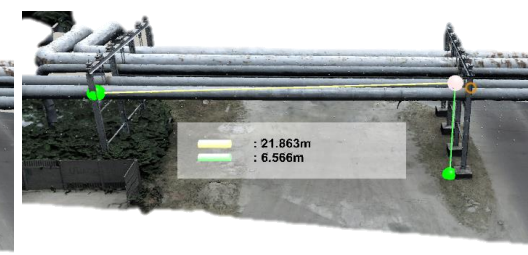

(c)

Figure 9. To take a measurement the user has to connect two markers placed near the model. (a) The user creates a connection between two points by gazing over the initial marker and making a pinch gesture (b). The user gazes over another marker and repeats the pinch gesture to generate a new ruler (b-c).

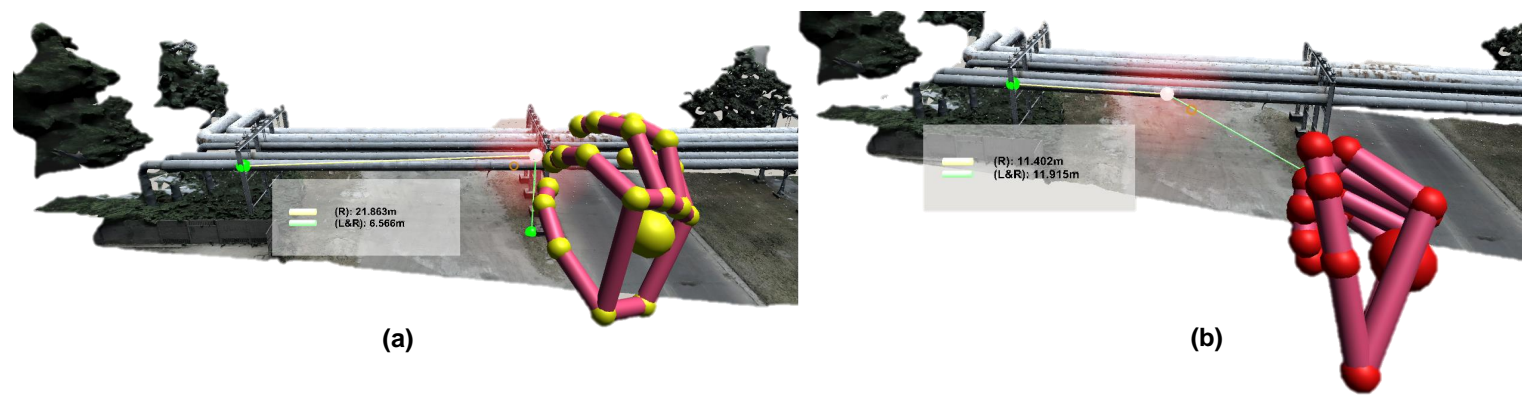

Figure 10. Once the markers are connected with rulers and their positions are changed $(a-b)$, the rulers will not disconnect themselves from the markers and will adjust their position and length. The rulers will automatically recalculate their respective lengths which are immediately visible in the HUD.

\begin{tabular}{|c|c|c|c|c|c|c|c|}
\hline & \multicolumn{3}{|c|}{ Measurements: Supporting Structure } & \multicolumn{3}{c|}{ Quantitative Data: Model Manipulation } \\
\hline Participant & Height & Width & Distance & Dislocation & Rotation & \multicolumn{2}{c|}{ Scale } \\
\hline number & {$[\mathrm{m}]$} & {$[\mathrm{m}]$} & {$[\mathrm{m}]$} & nominal units & {$[$ degrees] } & min & max \\
\hline P1 & 7.128 & 10.443 & 23.730 & 106.16 & $153.15^{\circ}$ & 0.004 & 1.479 \\
\hline P2 & 7.028 & 10.441 & 24.044 & 12.82 & $83.48^{\circ}$ & 0.002 & 0.050 \\
\hline P3 & 6.895 & 10.047 & 23.433 & 26.40 & $78.55^{\circ}$ & 0.004 & 0.300 \\
\hline
\end{tabular}

Table 1. The measurements taken by the participants and the extent to which the participants manipulated the pipe-rack model. The nominal scale of the model in relation to the real-life structure is 0.05 .

and thereafter we asked them to fulfill the following task: Please measure-using the interaction methods presented - the height of the supporting structure, and the distance between neighboring structures, as well as the width of one of them. Please take all other measurements that you consider relevant when surveying the asset.
The participants were given unlimited time to perform the task and instructed to think aloud. They were also observed, video- and audiorecorded and instructed to inform the researcher about any barriers they were facing when carrying out the task. When this task was completed, we had a semi-structured discussion with the 
participants.

These activities were aimed to give the participants an opportunity to learn the interaction methods while simultaneously providing them with a goal to focus on. We wanted to see how they were going to approach learning and using the interaction methods and how easy or how difficult they would find the entire process. Therefore we were not particularly concerned with very detailed measurements but rather with the overall user experience.

\section{Questionnaire and Survey Results}

We used established survey instruments to assess simulator sickness (SSQ) [6], flow short scale (FSS) [7], sense of presence (IPQ $)^{4}$, and perceived cognitive load (NASA TLX) [8]. For SSQ, P1 reported no symptoms while P2 and P3 reported mild symptoms. For FSS, P1-P3 all reported relatively high levels of flow but also high levels of anxiety. For IPQ, we observed average scores of 3.2/7 (P1), 3.6/7 (P2), and 3.1/7 (P3), respectively as we optimized our environment for comfort and ease concerning the measurements task. For NASA TLX, the participants reported relatively small levels of perceived cognitive load.

\section{Measurement Results}

All participants managed to obtain similar results within $\pm 0.5[\mathrm{~m}]$ precision (see Table 1 "Measurements"). P2 stated that, although it is hard to make general comments on the required measurements' accuracy (as it depends on the engineering purpose of the measurement), in the case of pipelines (...) even half a meter is enough [accuracy] and (...) no more is needed [based on his vast experience as an inspector, in this particular case] because it is quite a large pipe (...).

Participants also make use of model manipulation possibilities by dislocating, rotating or resizing it (see Table 1 "Quantitative Data").

\section{Discussion}

We analyzed the users' feedback and behaviors with particular attention to three perspectives: 1) how they interacted with the $3 \mathrm{D}$ model in VR;

\footnotetext{
${ }^{4}$ www.igroup.org/pq/ipq
}

2) the perceived usefulness of the measurement toolkit; and 3) suggestions and ideas for further system improvements.

\section{Model Manipulation}

The participants were satisfied with the implemented interaction methods for model manipulation. For example, P1 remarked that (...) what I like very much is (...) rotating, moving, bringing closer (...) [the model] and further, here I can see the entire pipeline, all suspensions but it does not give me the opportunity to focus on details. P2 commented that in terms of displacement, zooming in and out of the model it is OK. Our observations of the participants' behavior also suggest that all the participants promptly gained fluency in model manipulation.

\section{Measurement Toolkit}

One of the key aspects of the system was the snapping grid, which instead of being helpful, tended to confuse participants. For example, P1 commented that [snapping points] don't help me (...) they are confusing because they suggest something to me [which distracts him from his task]. P1 also remarked that if it was not for these [snapping] spheres, I could indicate more precisely the place I want [to put the marker at].

In general, even though all the participants (P1-P3) managed to capture the required measurements, they would prefer to obtain a higher accuracy when placing the markers. This possibility was constrained by the snapping radius being predefined in the interface, which increased proportionally to the model increase in size. It is possible to rectify this by either keeping the snapping radius small and constant, or by modifying how it responds to the model's enlargement.

P1 reported more interest in the pipeline details rather than in its measurements. P1 would, for example, like to inspect some discoloration observed on the pipe model with intense detail. P1 also desired additional data overlaid on the model, including localized temperature or pressure readings relevant to P1's current work duties. Similar remarks were shared by $\mathrm{P} 2$, who also commented that when using our system, the engineer does not have to be physically present on-site to take measurements. This means it is not limited by atmospheric conditions or health hazards caused 
by substances flowing through the pipes.

\section{CONCLUSIONS}

This paper has demonstrated the potential of VR-based systems to be useful in practical real-words engineering contexts. In addition, this paper has also demonstrated that populating VR immersive environments with photogrammetric models is a promising research trajectory for VR research in general.

The data analysis suggests a range of research avenues for future work, such as refining the hand-menu layout and the toolkit design. Another fruitful area for future investigations is to investigate the effectiveness of measurements of other real-world quantities of a structure, such as its size, surface area, or volume [5].

\section{ACKNOWLEDGMENT}

We would like to express our gratitude to Bentley Systems Inc. allowing the use of ContextCapture to generate the pipe-rack model. We also thank Krzysztof Kutt for verifying the questionnaire translations, and to Jerzy Sokołowski and Roman Górecki from the UDT Łódź for their aid with the user study.

This work was supported by the Cambridge Trust and the EPSRC (1788814).

\section{REFERENCES}

1. M. M. Thompson, "Manual of photogrammetry," Geoscience Abstracts, 1966.

2. A. El Saddik, "Digital Twins: The Convergence of Multimedia Technologies," IEEE MultiMedia, vol. 25, no. 2, pp. 87-92, Apr. 2018.

3. J. R. Wixson, "Function analysis and decomposistion using function analysis systems technique," in International Council on Systems Engineering Annual Conference, vol. 9, no. 1. Wiley Online Library, 1999, pp. 800-805.

4. S. K. Tadeja, P. Seshadri, and P. O. Kristensson, "Digital Twin Assessments in VR: An Explorational Study with Aeroengines," in Proceedings of the 41st IEEE Aerospace Conference, Big Sky, USA, Mar. 2020.

5. S. K. Tadeja, Y. L. Lu, M. Rydlewicz, W. Rydlewicz, T. Bubas, and P. O. Kristensson, "Exploring Gestural Input for Engineering Surveys of Real-life Structures in Virtual Reality Using Photogrammetric 3D Models," Multimedia Tools and Applications, 2021.
6. R. S. Kennedy, N. E. Lane, K. S. Berbaum, and M. G. Lilienthal, "Simulator Sickness Questionnaire: An Enhanced Method for Quantifying Simulator Sickness," The International Journal of Aviation Psychology, vol. 3, no. 3, pp. 203-220, Jul. 1993.

7. F. Rheinberg, R. Vollmeyer, and S. Engeser, "Assessment of flow experiences," Diagnosis of Motivation and Self-Concept (Tests and Trends), pp. 261-279, 2003.

8. S. G. Hart and L. E. Staveland, "Development of NASA Task Load Index: Results of Empirical and Theoretical Research," in Advances in Psychology. Elsevier, 1988, vol. 52, pp. 139-183.

Stawomir K. Tadeja is a research associate in the Department of Engineering at the University of Cambridge in Cambridge, UK. Contact him at skt40@cam.ac.uk.

Wojciech Rydlewicz is the COO of CS Softdesk in Łódź, Poland. Contact him at wojciech.rydlewicz@softdesk.pl.

Yupu Lu was a visiting student in the Department of Engineering at the University of Cambridge in Cambridge, UK. Contact him at y1737@cam.ac.uk.

Tomasz Bubas is a senior information specialist at CS Softdesk in Łódź, Poland. Contact him at tomasz.bubas@softdesk.pl.

Maciej Rydlewicz is the CEO of CS Softdesk in Łódź, Poland. Contact him at maciej.rydlewicz@softdesk.pl.

Per Ola Kristensson is a Professor of Interactive Systems Engineering in the Department of Engineering at the University of Cambridge in Cambridge, UK. Contact him at pok21@cam.ac.uk. 